\title{
STEM Z-contrast and Electron Energy Loss Spectroscopy Characterization of Co-doped $\mathrm{TiO}_{2}$ Thin Films
}

\author{
Lianfeng Fu, ${ }^{*}$ Nigel D. Browning, ${ }^{* * *}$ Shixiong Zhang, ${ }^{* * *}$ Darshan C. Kundaliya, ${ }^{* * *}$ Satish B. \\ Ogale, $^{* * *}$ and T. Venkatesan ${ }^{* * *}$ \\ * Department of Chemical Engineering and Materials Science, University of California at Davis, \\ Davis, CA 95616 USA \\ ${ }^{* *}$ National Center for Electron Microscopy, Lawrence Berkeley National Laboratory, Berkeley, CA \\ 94720 USA \\ *** Center for Superconductivity Research, Department of Physics, University of Maryland, College \\ Park, MA 20742, USA
}

Recently, Matsumoto et al reported the occurrence of room temperature ferromagnetism (FM) using a Co-doped anatase $\mathrm{TiO}_{2}$ thin film [1]. This work has generated great interests in the study of diluted magnetic semiconductor (DMS) materials, which are based on the semiconductors doping with low concentration dopants of 3d-group magnetic elements [2]. However, the fundamental issues in the DMS materials, such as the origin and the nature of FM, are still in controversy. Beside this, the magnetic properties of Co-doped $\mathrm{TiO}_{2}$ films are found to depend critically on the Co distribution, which is also strongly dependent on the growth process [3]. In this regard, the structural qualities of the diluted magnetic semiconductors play an important role in maintaining the spin character of the electron and its magnetic properties in the devices. To fully develop the DMS materials for spintronics applications, it is therefore necessary to characterize and understand the properties of the DMS thin films as a function of the growth conditions. It is well known that Z-contrast imaging in scanning transmission electron microscopy (STEM) can easily identify the hetero-interfaces with different compositions [4]. The use of EELS to determine the chemical composition and obtain other electronic information is also well established [5]. Therefore, the conjunction of Z-contrast imaging and EELS analysis is very useful for the microscopic characterization of thin films.

Thin films of anatase $\mathrm{Ti}_{0.97} \mathrm{Co}_{0.03} \mathrm{O}_{2}$ studied in this work were grown on (001) $\mathrm{LaAlO}_{3}(\mathrm{LAO})$ single crystalline substrates by a pulsed laser deposition method with an excimer laser at $875{ }^{\circ} \mathrm{C}$ at an oxygen partial pressures of $1 \times 10^{-5}$ torr. After the growth, the cross-section of thin film samples was characterized using STEM Z-contrast and electron energy loss spectroscopy (EELS) on a 200kV Schottky field-emission gun (FEG) FEI Tecnai F20. Core-loss EEL spectra were recorded with a dispersion of $0.2 \mathrm{eV} /$ channel to contain more energy windows and an exposure time of $6 \mathrm{~s}$. The background for each core-loss spectrum was subtracted by a power-law fitting method.

A typical STEM Z-contrast micrograph for the $\mathrm{Ti}_{0.97} \mathrm{Co}_{0.03} \mathrm{O}_{2}$ thin film sample is shown in Fig. 1a. The micrograph is deliberately shown on a coarser length scale to check the presence or absence of any distributed clusters. In the micrograph, some nanoclusters with the size of 10-60 nm were observed to form mostly at the heterointerface. The distribution of $\mathrm{Co}$ in the film and the composition of clusters were studied by probing the film via tens of EELS scans across the sample. A typical such series of EELS scans is presented in Fig. 1b. The white lines intensity ratio for the Co $\mathrm{L}_{2,3}$-edge have been observed at $795 \mathrm{eV}$ and $780 \mathrm{eV}$ respectively implying an oxidized state of Co at 2.3+. Higher resolution STEM Z-contrast imaging results (not shown here) show the structural details of nanoclusters including the existence of stacking faults. The atomic structure of 
nanoclusters was shown to be similar to that of $\mathrm{TiO}_{2}$ matrix, except the Z-contrast intensity change between them. This means that the clusters are composed of heavier elements than $\mathrm{Ti}$ in an anataselike structure. These experimental results showed that the clusters were probably Co-rich titanium oxide and were not the metallic Co usually observed in Co-doped $\mathrm{TiO}_{2}$ films. Moreover, the interface was the preferential nucleation sites for these nano-clusters due to the low interfacial energy and instability. We have seen earlier [6,7] that small changes in the nominal growth conditions examined here can lead to a fairly uniform cobalt Incorporation in $\mathrm{TiO}_{2}$ matrix (anatase and rutile) at low Co concentration. This work thus strongly suggests that the Co distribution in $\mathrm{TiO}_{2}$ thin films was sensitive to the oxygen pressure and growth conditions. The formation of Co rich $\mathrm{TiO}_{2}$ clusters should be the main origins for the observed FM in this film.

\section{References:}

[1] W. Prellier et al , J. Phys.: Condens. Matter., 15 (2003) 1583.

[2] Y. Matsumoto et al, Science, 291 (5505) (2001) 854.

[3] J.K. Furdyna, J. Appl. Phys., 64 (1988) 29.

[4] L.F. Fu et al., Appl. Phys. Lett. 87 (2005) 262904.

[5] N.D. Browning et al., Nature 366 (6451) (1993) 143.

[6] T. Zhao et al. Phys. Rev. Lett. 94 (2005) 126601.

[7] S. X. Zhang et al., Appl. Phys. Lett. 88 (2006) 012513.

[8] This work was performed at the NCEM, Lawrence Berkeley National Laboratory and supported by DOE Contract No. DE-AC02-05CH11231, NSF Grant No. DMR-0335364, DMR-0520471, and DARPA Spin S program, NSF-MRSEC Grant No. DMR-00-80008.

(a)

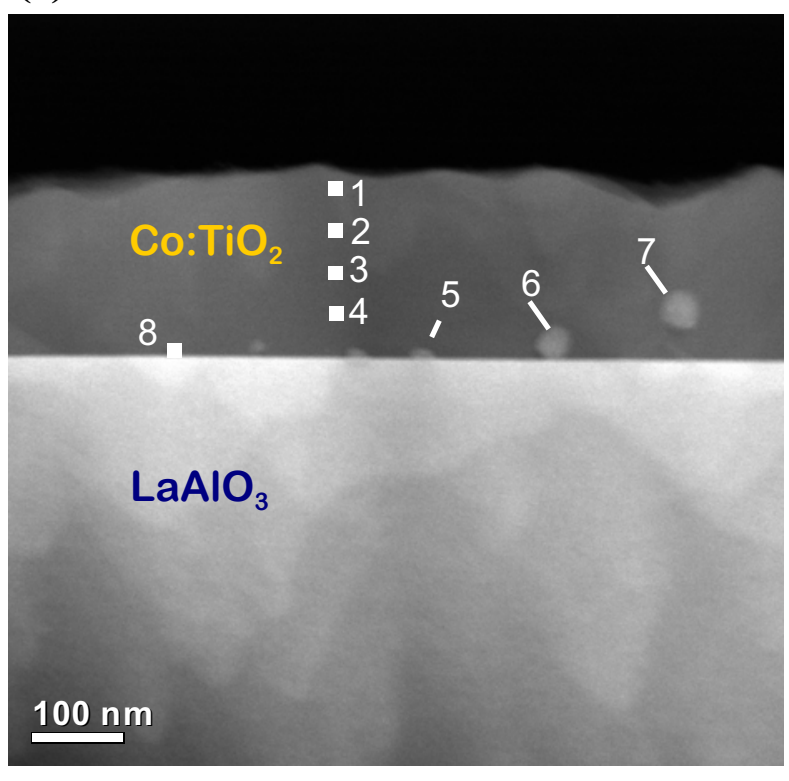

(b)

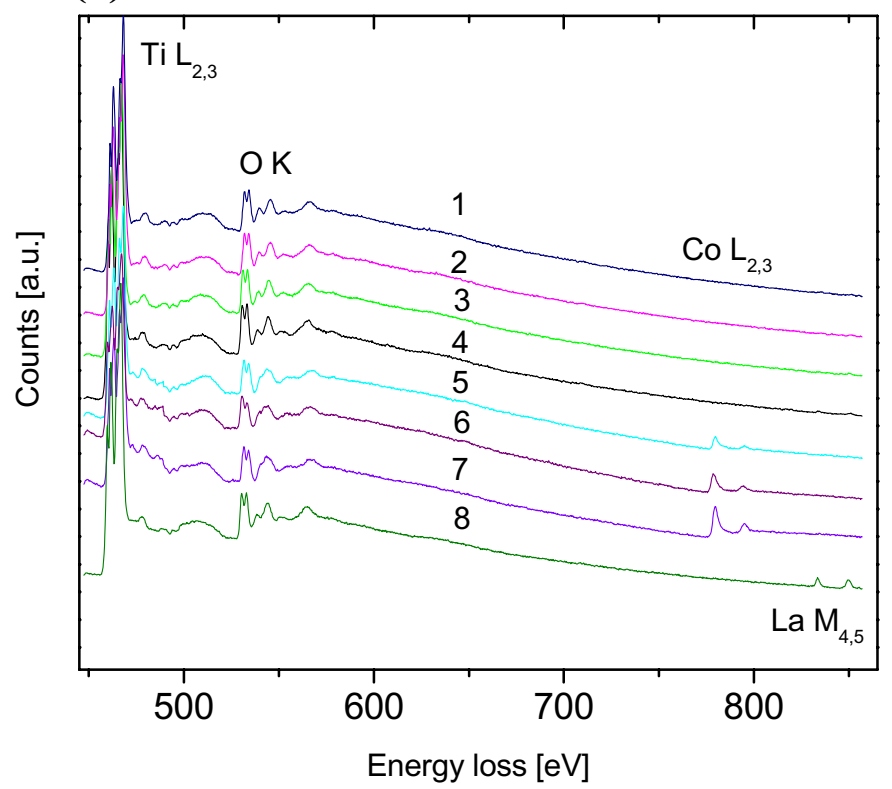

Fig. 1. (a) a cross-sectional STEM Z-contrast image of the Co-doped $\mathrm{TiO}_{2}$ films showing the existence of nanometer-size clusters (b) EEL spectra at $\mathrm{Ti} \mathrm{L}_{2,3}$ edges, $\mathrm{O} \mathrm{K}$ edges, $\mathrm{Co}_{2,3}$ edges, and La $\mathrm{M}_{4,5}$ edges acquired from the corresponding points shown in Fig. 1(a). The strong signal in $\mathrm{Co}$ $\mathrm{L}_{2,3}$ edges shows the aggregation of Co elements in the nanometer-size clusters. 